\title{
Small Molecules Polypharmacology for the Treatment of Breast Cancer: A Roadmap for the Future Chemotherapy
}

\author{
Reetuparna Acharya, Shakti Prasad Pattanayak* \\ Department of Pharmaceutical Sciences and Technology, Birla Institute of Technology, Mesra, Ranchi, Jharkhand, INDIA.
}

\begin{abstract}
Background: Breast cancer is considered as the most common ailment in women across the globe. There are a variety of major factors for the growth and progression of breast cancer. Although genetic changes are key factors for cancer progression, epigenetic and environmental factors also have their fair share of contribution. Hypothesis: An attempt has been made in the review to highlight about small molecule polypharmacology for the treatment of breast cancer which is the design of small molecules (molecular weight $<900 \mathrm{Da}$ ) that aim for two or more cancer targets (tyrosine kinases). Small molecules are advantageous over conventional chemotherapy with higher target specificity, lesser known side effects, simplified dosing schedule, non-invasive dosing and improved patient compliance. Results and Conclusion: Small molecules have been introduced since the past two decades and has been a potential success in the field of cancer chemotherapy, but there are lot more to explore. The review mainly focuses on the essential cellular signaling pathways involved in breast cancer progression and the recent small molecule inhibitors that show promising activity against the pathways.

Key words: Breast cancer, Small molecules, Polypharmacology, Tyrosine kinases, Chemotherapy.
\end{abstract}

\section{INTRODUCTION}

Breast cancer is considered as the most common ailment amongst women in both developing and developed countries. As per GLOBOCAN statistics (2012), amongst $25 \%$ (1.67 million) of new cases of cancer, breast cancer caused 15\% (522,000) of all cancer deaths in women. ${ }^{1}$ Breast cancer being a heterogeneous disease shows a great extent of tumor complexity. It is therefore needed to elicit a better response with treatment strategies in patients. ${ }^{2}$ According to the profiling of gene expression, breast cancer has been classified into four types: luminal A, luminal B, basal-like breast cancer and HER-2 overexpressed breast cancer. Luminal A being the most common type consists of ER and/or PR positive, HER-2 negative and $\mathrm{Ki}-67$ expression less than $14 \%$. Luminal $B$ is considered the second most common consisting of ER and/or PR positive, HER-2 negative and $\mathrm{Ki}-67$ greater than or equal to $14 \%$. Basal like which is also known as triple negative breast cancer (TNBC) has ER, PR and HER-2 negative genes. HER-2 overexpressed type is uncommon and consists of ER, PR negative and HER-2 overexpression. ${ }^{3}$ The mentioned classification of breast cancer varies in their phenotypes which can prognose for the drug response, survival rates and treatment strategies. ${ }^{4}$

TNBC or basal like breast cancer is characterized by high grade metastatic tumors which affects lymph nodes much more than the other subtypes. It also comes with a poor prognosis of progression free and/or overall survival. ${ }^{5}$ Although basal-like breast cancer has a poor prognosis, the main form of treatment is systemic chemotherapy. The other subtypes of breast cancer (luminal A,
Submission Date: 31-08-2019; Revision Date: 05-11-2019; Accepted Date: 26-11-2019

DOI: 10.5530/ijper.54.2.27 Correspondence: Dr. Shakti Prasad Pattanayak,

Department of Pharmaceutical

Sciences and Technology, Cancer Research Group, Division of Pharmacology, Birla Institute of Technology, Mesra, Ranchi-835215, Jharkhand, INDIA. Phone: +916512275444 E-mail: sppattanayak@ bitmesra.ac.in

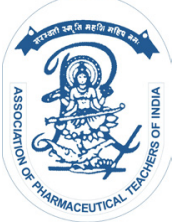

www.ijper.org 
luminal B and HER-2 overexpressed) are treated with targeted therapy. ${ }^{6}$ In this review, we try to highlight the chemical classification of small molecules in the treatment of breast cancer along with their multi target effects on various proteins in molecular cellular signaling pathways of breast cancer initiation and progression.

\section{Chemotherapy of Breast Cancer}

Chemotherapy made its way between 1960-1980 where Tamoxifen (1971) was introduced as a potent ER inhibiting agent Then, the first rationalized targeted therapy for breast cancer came with the development of Trastuzumab, which is a humanized form of anti-p185 HER-2 monoclonal antibody. Being highly HER-2 specific, Trastuzumab showed higher benefits in case of breast cancer with elevated HER-2 levels. ${ }^{7}$ Taxanes (Paclitaxel, Docitaxel, Nab-Paclitaxel) were introduced in the 1990s and Paclitaxel was marked as a potent agent for the treatment of Metastatic Breast Cancer (MBC). The various treatment options for stagewise breast cancer is given in Table 1. The recent upcoming treatment options for breast cancer include Urokinase plasminogen activators and plasminogen activator inhibitors-1 which are considered as new prognostic markers. The decrease in the concentration of markers predicts that there will be a decrease in cancer recurrences. ${ }^{8}$ Also, the expression of 21 genes (genes selected from published literature of cancer patients, databases and gene expression finding that had 5 control and 16 cancer associated genes) is measured by an oncotype DX assay which gives the prediction whether chemotherapy will prove to be less

\begin{tabular}{|c|c|c|c|}
\hline \multicolumn{3}{|c|}{ Table 1: Chemotherapeutic drugs used for different } \\
stages of breast cancer.
\end{tabular}

beneficial for node negative patients. ${ }^{9}$ PARP (Poly ADP Ribose Polymerase) inhibitors are introduced as potent anti-cancer agents which are small molecules that mimic the action of nicotinamide that binds irreversibly to the NAD + site of PARP 1 and 2, hereby hindering PARylation and leading to DNA repair. PARP inhibitors, Olaparib, Talazoparib, Veliparib, Ipatasertib, Buparlisib, Atezolizumab, Durvalumab, Pablociclib have been clinically tested in Hormone Receptor (HR) Positive (ER and PR positive) breast cancers. Histone deacetylation mediates the loss of ER expression in breast cancer which causes hormonal resistance in ER positive patients. Histone Deacetylase (HDAC) inhibitors are used to block the activity of the enzyme, upregulate ER $\alpha$ expression and block growth factor signaling pathways. Entinostat and Vorinostat are the HDAC inhibitors used in breast cancer chemotherapy. Studies reported that expression and activity of steroid sulfatase was highly increased in breast cancer patients. Steroid sulfatase is an enzyme that is involved in the conversion of sulfate conjugated enzymes (inactive form) into estrogenic non-conjugate from (active). Other advances include the use of monoclonal antibodies (MAbs): Patritumab (anti-HER-3 MAb), Margetuximab (Anti-HER-2 MAb) and Pembrolizumab, farsenyl transferase inhibitors (Lonafarnib) that prevents Ras activity by farsenylation.

\section{Small Molecules Polypharmacology in Breast Cancer}

A small molecule is an organic compound having a molecular weight less than 900 Daltons. ${ }^{10}$ Small molecules are becoming an emerging trend for the treatment of cancer as they provide enhanced target specificity due to their small size and low molecular weight. They can easily target the extracellular proteins, cell surface ligand-binding receptors and also the intracellular proteins that play a vital role in the antiapoptotic pathways. Small molecules have been reported to target serine/threonine/tyrosine kinases that are critical cancer targets. Other proteins that are reported to be targeted by small Molecules are Metalloproteinases (MMPs), proteasomes, Heat Shock Proteins (HSPs) and proteins playing part in signal transductions. ${ }^{11}$ A certain example of small molecule comes under the class of tyrosine kinase inhibitors. Protein kinases are a family of enzymes that phosphorylate their substrates. They are classified into three major types: Serine/threonine kinases, tyrosine kinases and dual specificity protein kinases. The first type of kinase phosphorylates either a serine or threonine amino acid, the second type phosphorylates the kinase amino acid and the third type can phosphorylate both residues. ${ }^{12}$ Kinases are recognized as critical targets for cancer because of their 
vital role in the regulation of cellular growth, metabolism, differentiation, migration and survival. The ERBB family belongs to receptor tyrosine kinases which consists of 4 subgroups: EGFR/HER1 (Epidermal Growth Factor Receptor), ERBB2/HER2, ERBB3/ HER3 and ERBB4/HER4. These phosphorylated proteins have the capability to trigger certain downstream regulatory pathways: PI3K/Akt (Phosphoinositide-3kinase) and Akt also known as PKB (Protein Kinase B), Ras/MEK/ERK, JAK (Janus Kinases)/STAT (Signal Transducer and Activator of Transcription Proteins) and PLCy (Phospho Lipase C)/PKC (Protein Kinase C). These pathways have a major act in all sorts of cellular processes such as cell survival, regulation, differentiation, proliferation, angiogenesis, motility, apoptosis, invasion and adhesion. ${ }^{13}$ The schematic diagram of breast cancer pathways with their downstream signaling is shown in Figure 1 and the cascade of apoptosis inducing reactions and the effect of small molecules on them are shown in Figure 2. These pathways are also responsible for the

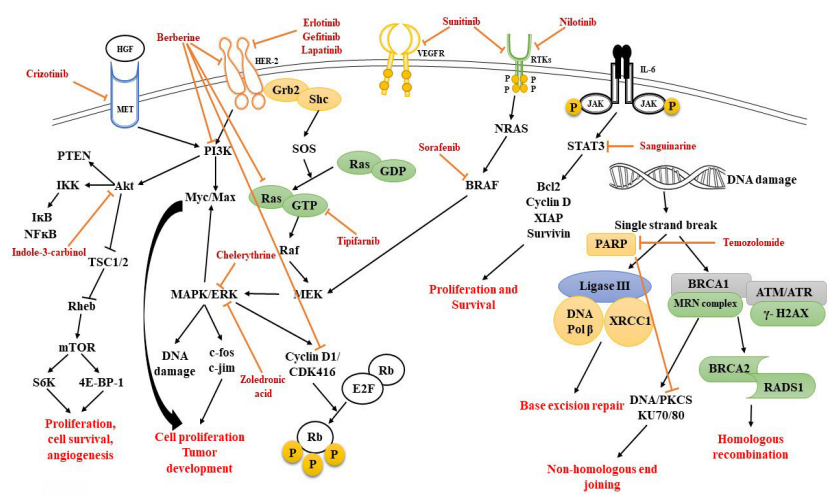

Figure 1: Summarized small molecules targeted therapy effective against breast cancer. Schematic representation of breast cancer progression pathways along with small molecule inhibitors against the critical targets.

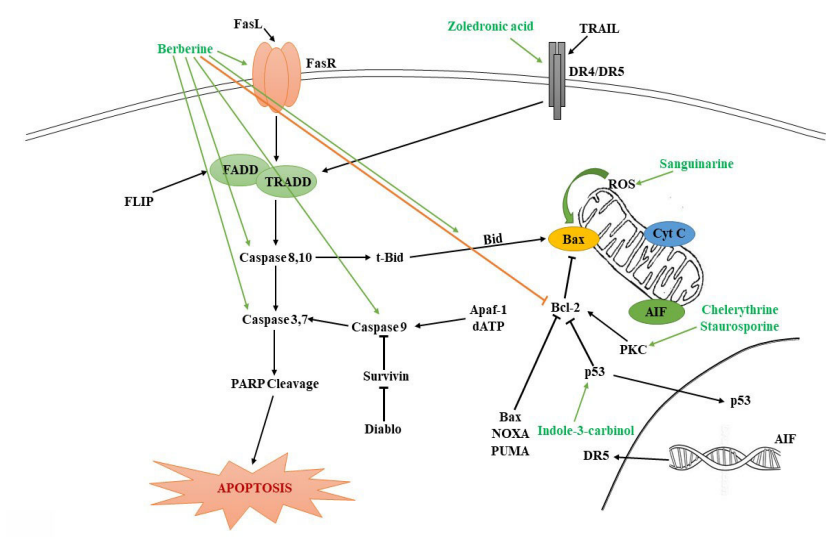

Figure 2: Schematic diagram of cascade of reactions taking place for apoptosis along with the small molecules having apoptosis inducing activity in cancer cells. upregulation of epithelial mesenchymal transition, a gene that marks the beginning of carcinogenesis, invasion and metastasis. ${ }^{14}$ Out of all the different types of ERBB family subgroups, EGFR and HER2 are commonly overexpressed in many forms of cancer, such as breast cancer, hence drawing attention as an important target against cancer. ${ }^{15}$ The small molecule receptor tyrosine kinase inhibitors act by competing with ATP in binding to the kinase domain of the receptor. ${ }^{16}$ This prevents the autophosphorylation of the receptor which activates intracellular functions. ATPs are present in higher concentrations in the cell so in order to function efficiently, small molecules must be administered in higher doses. ${ }^{17}$ Also, small molecules have the privilege of lesser invasive administration route as they are orally active compounds. ${ }^{18}$

Combination chemotherapy consists of more than one drug regimens to target multiple cancer pathways but there are a lot of problems faced along with it, patient incompliance being the major one. The non-specificity in cancer targets leads to higher toxicity as the drugs act on normal as well as cancer cells. Also, most of the chemotherapeutic agents are given intravenously and are given in combinations which makes them tiresome and highly patient incompliant. To overcome with the situation, small molecules can be designed in a manner by which multiple cancer targets can be aimed for the prevention and improved prognosis of cancer. Polypharmacology is the term coined for the designing of a single molecule that is capable of interacting with multiple targets simultaneously and specifically. "Small Molecule Polypharmacology (SMPP)" is the design of a compound that will have a molecular weight less than 900 Daltons with more than one critical cancer targets. There are several advantages of polypharmacology over combination chemotherapy.

Polypharmacological agents have better efficacy towards their targets in lethal diseases, like cancer. The pharmacokinetic profiles of these agents come with improved prediction level and safety profile. Moleculebased adverse effects can be overcomed by implementing polypharmacology as there is a single molecule. Toxicity of treatment can be reduced to an impressive extent as there are no multiple drug regimens showing adverse drug interactions and negative synergistic actions. The intensity of development of target-based resistance is statistically decreased in polypharmacology when compared to single-target drugs. Dosing schedules are simplified and improved patient compliance is achieved comparatively to combination therapies, especially in case of chronic diseases like cancer. There is a possibility of decreased length of treatment due to 
higher efficacy and target achievement of drug with a decrease in cost of drug due to lesser developmental issues. If we focus on drug formulation manufacturing process, there will be an ease in the evaluation of pharmacokinetic and pharmacodynamic properties of the molecule because of it being a single chemical entity rather than being a combination. Also, the manufacturing cost will be decreased as a single entity is easier to formulate than a mixture. ${ }^{20}$ The concept of polypharmacology is relatively young and has grown massively in the last decade, specifically in the treatment of cancer and depression. The success has drawn attention towards single molecules with multiple targets which are compatible with both safety and efficacy issues. ${ }^{21}$

\section{Chemical Classification of Small Molecules: Potential to have Anti-Breast Cancer Activity:}

(i) Quinoline derivatives: Quinoline is an aromatic, nitrogen-containing compound which has pharmacologically proven activities like anti-bacterial, antifungal, anti-malarial, anti-convulsant, analgesic and anti-inflammatory. ${ }^{22}$ Quinolines have been extensively studied for anti-cancer action and has been reported to show competitive binding activity towards ER ${ }^{23}$ Quinolines have also been reported to possess selective cyclooxygenase (COX-2) inhibition activity and has been tested to have in-vitro anti-breast cancer activity by inhibition of phosphoinositide specific phospholipase Cy enzymes by arresting the cell cycle at G2/M phases. $^{24}$ Studies also reported aromatase and aldosterone synthase inhibition potential by quinolone compounds. ${ }^{25} \mathrm{~A}$ few examples of quinoline compounds which are effective against breast cancer are Berberine, Camptothecin, Chelidonine, Chelerythrine, Lavendamycin, Nitidine and Sanguinarine. ${ }^{26}$ Berberine is a small molecule having a molecular weight of 336.36 Daltons which shows multitargeted anti-cancer action. It inhibits HER-2 receptor action which has a key role in breast cancer development. It also acts on proteins, PI3K, Ras, Raf-1, MEK and cyclin D1 which are important proteins involved in cell signaling pathways. ${ }^{27}$ Berberine induces apoptosis, both in a caspase independent manner that increases ROS level by JNK/p38 MAPK signaling pathway and also by the activation of caspases-3,8 and 9 by the expression of both Fas and FasL. Berberine also has a role in the deactivation of two major caspase inhibitors, c-IAP1 and XIAP hereby, promoting apoptosis. ${ }^{28}$ Camptothecin is a plant alkaloid that targets the enzyme, DNA topoisomerase I (enzyme required for DNA single strand breakage and reduction in supercoiling). Camptothecin shows S-phase specific cytotoxicity by formation of covalent cytotoxic
camp-top1-DNA intermediates. Accumulation of this intermediate (camp-top1-DNA) activates the pathways related to DNA damage and leads to DNA damage. ${ }^{29}$ Chelidonine is an isoquinoline compound which forms as a secondary metabolite in the source plant and has shown potent anti-cancer potential in a variety of malignancies (gastric, breast etc.). Chelidonine has been reported to overexpress p53 onco-suppressor gene which initiates apoptosis and autophagy. ${ }^{30}$ Chelerythrine is an alkaloidal drug isolated from a herb and has been reported to show potent anti-tumor effect against breast, colon and prostate cancers. It shows inhibitory action for protein synthesis and cell cycle progression. It also activates signaling pathways such as JNK/p38 MAPK and MEK/ERK1/2 leading to apoptosis induction in cancer cells. It has also been reported to cause bimodal cell death and G1-phase cell cycle arrest in leukemia cells. ${ }^{31}$ Nitidine is a naturally occurring quinoline compound which has proven actions like anti-tumor, anti-leukemic, anti-inflammatory and anti-HIV. Nitidine has been reported to show anti-cancer activity by decreasing cell growth, apoptosis induction, cell-cycle arrest and DNA ligase activity inhibition in cancer cells. In recent research, it was also proven that Nitidine blocked c-Src/FAK signaling pathways hereby, inhibiting the activity of matrix metalloproteinases, MMP-9 and MMP-2. ${ }^{32}$ Sanguinarine is an alkaloidal compound that has displayed cardiac, anti-tumor and antihypertensive actions. Sanguinarine has proven action against breast cancer which shows that it has TPA induced MMP-9 and COX-2 expression which is caused by the blocking of $\mathrm{NF}_{-\mathrm{K}} \mathrm{B}, \mathrm{Akt}$ and ERK1/2 signaling pathway. It also inhibited that migration and invasion of breast cancer cells where the anti-invasive action was due to induction of HO-1 expression. ${ }^{33}$

(ii)Quinazoline derivatives: Quinazolines are heterocyclic compounds with a benzene ring fused with pyrimidine ring and are known to have various pharmacological activities such as sedative, hypnotics, anti-convulsant, anti-inflammatory, analgesic, anti-cancer, anti-microbial, anti-viral, anti-tubercular etc. ${ }^{34}$ They are reported to show anti-neoplastic action by folate metabolism inhibition, tyrosine kinase inhibition, induction of apoptosis and by blocking topoisomerase function..$^{35} \mathrm{~A}$ few examples of small molecule quinazoline derivatives that are active against breast cancer are: Erlotinib, Lapatinib, Gefitinib, Canertinib and Dasatinib. Erlotinib is an orally active small molecule which has EGFR tyrosine kinase inhibitory action. Erlotinib functions by blocking certain signal transduction pathways essential for cancer cell survival and proliferation. It also causes 
cell cycle arrest in the G1 and S phases. ${ }^{36}$ Lapatinib is a small molecule dual tyrosine kinase inhibitor which has oral activity against breast cancer cells. It targets for both EGFR and HER-2 receptors and inhibits their downstream cell signaling pathways. ${ }^{37}$ Gefitinib is also an active small molecule which possesses reversible action against EGFR tyrosine kinase. It autophosphorylates the EGFR hereby, blocking its downstream pathways. ${ }^{38}$ Canertinib is a small molecule irreversible inhibitor of tyrosine kinase which was designed to inhibit all the four pan-ErbB family members providing it better specificity than single ErbB inhibitors. It binds covalently with the specific cysteine residues in the ATP-binding pockets of EGFR and inhibits their kinase activity and downstream signaling. ${ }^{39}$ Dasatinib is an orally acting small molecule tyrosine kinase inhibitor which has its action against various TKs such as ABL, Src, c-KIT, PDGFR- $\alpha$, PDGFR- $\beta$ etc. It is an approved molecule for the treatment of Acute Myeloid Leukemia (AML). Studies showed that Dasatinib inhibited both ABL and Src TKs in breast cancer patients and also showed its effect on STAT5, platelet derived growth factors and c-KIT. ${ }^{40}$

(iii) Pyridine derivatives: Pyridines are heterocyclic compounds that have reportedly shown anti-cancer activities. ${ }^{41}$ According to research, non-fused pyridines tend to show better actions, like inhibition of VEGFR-2 and initiation of Akt and eNOS, hereby suppressing VEGF dependent migration and proliferation. ${ }^{42} \mathrm{~A}$ few examples of small molecule pyridine molecules which used as effective anti-cancer agents are Sorafenib, Regorafenib, Vismodegib and Crizotinib. ${ }^{43}$ Sorafenib and Crizotinib have been reported to show anti-breast cancer potential. Sorafenib is a multi-kinase inhibitor which has inhibitory potential against VEGFRs (vascular endothelial growth factor receptors), PDGFRs (platelet derived growth factor receptors), serine/ threonine kinases and suppression of angiogenesis and tumor cell proliferation. Crizotinib is an orally active small molecule tyrosine kinase inhibitor that has shown potent anti-angiogenetic, anti-proliferative and cytotoxic activities in various malignancies. Crizotinib has been reported to inhibit ALK, MET and ROS-1 kinases. ${ }^{44}$ Studies showed that $20-30 \%$ of breast cancers have an elevated level of MET (receptor of HGF-Hepatocyte growth factor) and shows poor prognosis of disease in patients will lesser survival rates. ${ }^{45}$

(iv)Imidazole derivatives: Imidazole is an aromatic alkaloid and diazole with proven anti-cancer potential. Imidazole compounds have also reported to show many other pharmacological activities like anti-tubercular, anti-fungal, anti-bacterial, anti-parasitic, anti-viral, anti-obesity, anti-neuropathic, anti-hypertensive, antiinflammatory, etc. Some examples of small molecule imidazole compounds which have reported activity against breast cancer are: Temozolomide, Zoledronic acid, Mercaptopurine, Nilotinib and Tipifarnib. ${ }^{46}$ Temozolomide is an orally bioavailable imidazole derivative molecule which has alkylating activity and has the capability to cross blood brain barrier (hence, also used in brain tumor treatment). The drugs that are used for the treatment of metastatic breast cancer (Capecitabine, Vinorelbine, Herceptin, Gemcitabine and Trastuzumab) does not penetrate the blood brain barrier and has no role in prevention of breast cancer metastasizing into the brain. Temozolomide functions by deactivating the enzyme O6-alkylguanine DNA alkyltransferase (MGMT) which is a DNA repair enzyme. Breast tumors treated by Temozolomide have been found to have high levels of MGMT so as to repair the crosslinking caused by alkylation. ${ }^{47}$ It also plays a role in the inhibition of PARP (enzyme catalyzing Nicotinamide Adenine Dinucleotide $\left(\mathrm{NAD}^{+}\right)$dependent ADP-ribosylation of DNA. PARP also has a role in the activation of BER which prevents double strand breaks along with direct prevention of double strand breaks. ${ }^{48}$ PARP expression is elevated in the presence of various cases of malignancies. PARP inhibitors act as potent catalytic inhibitors by competing with $\mathrm{NAD}^{+}$for PARP substrate binding site. ${ }^{49}$ Zoledronic acid is a nitrogen containing imidazole which has osteoclast dependent bone resorption inhibitory activity. Breast cancer metastasis is mediated by dysregulation of mitochondrial dysregulation and TGF- $\beta /$ SMAD signaling. It might also be related to hypoxia which results in tumor progression and resistance from chemotherapy. ${ }^{50}$ Zoledronic acid was reported to inhibit ERK1/2 phosphorylation both in vivo and in vitro in breast cancer cells. It was also found to enhance the activity of antiestrogen therapy in ER positive breast cancer patients by suppressing HIF-1 $\alpha .{ }^{51}$ Nilotinib has been reported to show anti-cancer activity better in combination with antibodies. Nilotinib showed suppression of vessel maturation. It also showed in vivo anti-angiogenic potential but that was not due to direct effect of the drug on the tumor epithelium but rather because of indirect effect on the tumor stroma/cells. ${ }^{52}$ Tipifarnib is an orally active small molecule that is a farsinyl transferase competitive inhibitor. Farsenyl transferase inhibitors function by the inhibition of Ras pathway which leads to blockade of cancer cell proliferation. Tipifarnib suppressed the expression of HIF-1 $\alpha$ in the protein and mRNA levels and their following downstream genes (LDHA, PDKI, Glut 1, Snail). ${ }^{53}$ 


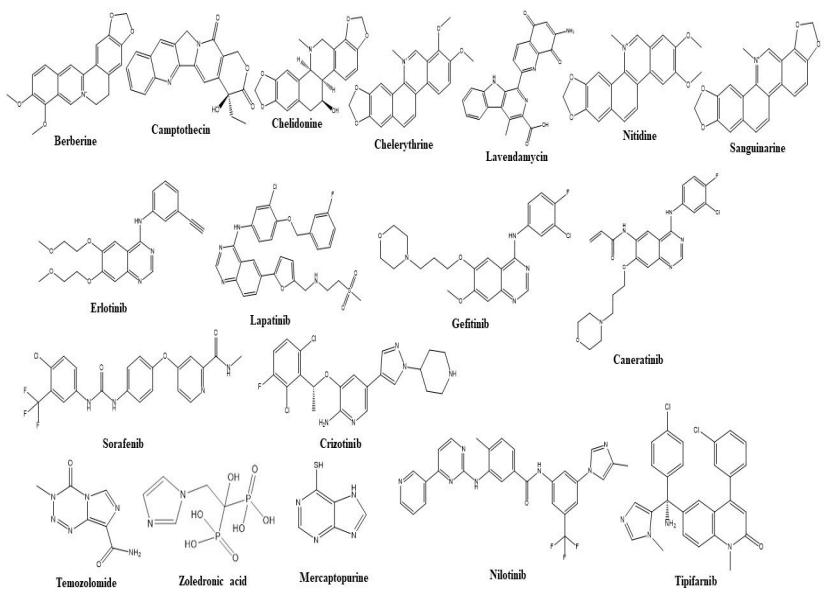

Figure 3: Chemical structures of Quinoline, Quinazoline, Pyridine and Imidazole derivative small molecules used in the treatment of breast cancer chemotherapy.

The chemical structures of the mentioned Quinoline, Quinzoline, Pyridine and Imidazole derivatives are shown in Figure 3.

(v)Indole derivatives: Indoles are aromatic heterobicyclic compounds having a six membered ring fused to a five membered pyrrole ring. ${ }^{54}$ Naturally occurring indoles function as signaling molecules in plants. Its synthesis is done by Fischer indole synthesis which is the oldest method for indole synthesis. ${ }^{55}$ Indoles have been reported to show anti-cancer potential in many studies when properly substituted. It was first isolated from Catharanthus roseus (vincristine, vinblastine) and has shown anti-cancer effects as tubulin targeting properties ${ }^{56}$ Some of the naturally occurring indoles having anti-cancer action are Indole-3-carbinol (I3C), 3',3'-Diindolylmethane (DIM) and Ascorbigen which have Akt and NF-kB dependent signaling inhibitory potential. Small molecule indole compounds active against breast cancer are Staurosporin, Sunitinib, Enzastaurin, I3C and DIM. Staurosporin is a potent apoptosis inducing compound which showed decreased expression of anti-apoptotic protein $\mathrm{Bcl}-2$ which led to the elevation of pro-apoptotic proteins $(\mathrm{Bax} / \mathrm{Bcl}-\mathrm{XS})$ in a time dependent manner. ${ }^{57}$ Sunitinib is a small molecule kinase inhibitor which has the potential to inhibit the potent mutant kinase, KIT along with VEGF receptors. ${ }^{58}$ Enzastaurin is an acyclic bisindolylmaleimide that competes for ATP binding site of PKC hereby, preventing substrate phosphorylation. Enzastaurin has been reported to inhibit tumor growth and angiogenesis in both in vitro and in vivo preclinical models. It has been found to have most potent action against PKC- $\beta$ and has shown potent endothelial cell proliferation inhibitory potential as compared to tumor cell proliferation

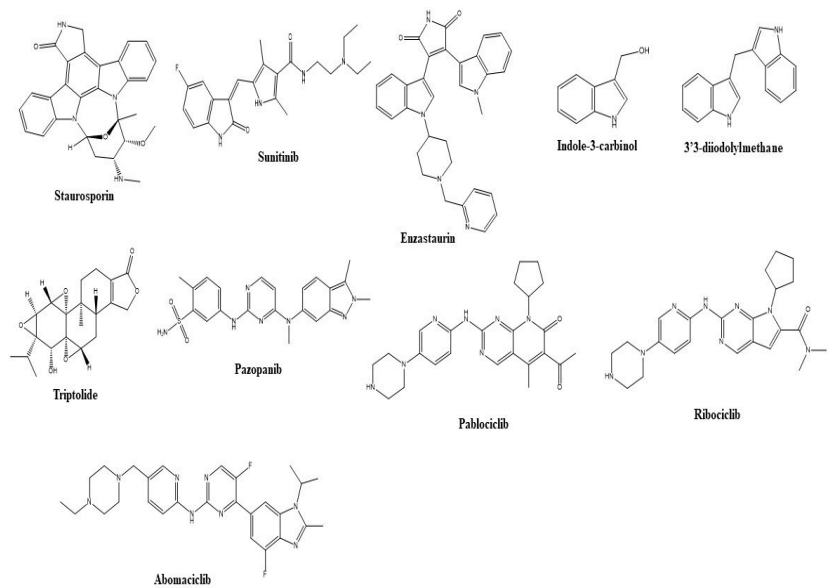

Figure 4: Chemical structures of Indole derivative small molecules and other miscellaneous small molecules used in breast cancer chemotherapy.

inhibition. ${ }^{59} \mathrm{I} 3 \mathrm{C}$ is a natural indole compound which is an indole glucobrassicin hydrolytic metabolite that has reported activity of proliferation suppression and apoptosis induction. It also possesses pleiotropic effects on cell signaling and physiology. DIM is a metabolite of I3C and both the indoles have inhibitory potential for Akt and NF-kB dependent signaling. I3C and DIM has also been reported to activate AhR which suppresses estrogen induced proliferation through transcriptional downregulation and proteasome degradation of ER $\alpha$. I3C also activates AhR and increases CYP450 (CYP1A1 and CYP1A2) expression that alters estrogen metabolism which decreases breast cancer risk. There are other miscellaneous small molecule inhibitors that are active against breast cancer: Triptolide, Pazopanib, Pablociclib, Ribociclib and Abomaciclib. The chemical structures of the mentioned Indole derivatives and some miscellaneous small molecule inhibitors are shown in Figure 4.

\section{Delivery of Small Molecules to target site of cancer}

Although, small molecules have better target reaching ability by translocating in the cellular plasma membranes in comparison with larger molecules but their target specificity can be further enhanced by incorporating them into certain delivery systems. Delivery systems also help in the management of poor bioavailability and toxicity issues. ${ }^{60}$ Incorporating the small molecules into a delivery system allows the drug for a controlled release, gives protection against degradation and decreases uptake of drug non-specifically to undesirable tissues. ${ }^{61}$ Some of the examples of delivery systems in which small molecules can be incorporated are nanoparticles, layer-by-layer nanoparticles, liposomes and Small Molecules Drug Conjugates (SMDCs). For 
making EGFR as a target for the treatment of breast cancer, GE11 peptides are conjugated with PEGylated polylactic-co-glycolic acid (PLGA) in the form of nanoparticles. This has been implemented on plant derived small molecule, curcumin and has been tested against breast cancer cell line EGFR expressing MCF-7 cells (in-vitro) and also in in-vivo studies. ${ }^{62}$ Layer-by-layer nanoparticles serve as a clinically potential delivery system by utilizing sequentially deposited oppositely charged polymers by building an extreme stable film with siRNA content in a nano sized core. This can be further engineered for better tumor targeting and modulating desired drug release from the core that has an enhancing effect on bioavailability. Layer-by-layer nanoparticles are a tunable multi drug delivery device. ${ }^{63}$ Liposomes are another choice for small molecule delivery. They are phospholipid bilayer vesicles that surrounds and aqueous compartment and encapsulates both lipophilic and hydrophilic molecules. ${ }^{64}$ Small Molecule Drug Conjugates (SMDCs) are an established perspective for the delivery of small molecules into the tumor site. SMDCs have the privilege of being non-immunogenic in nature, lesser molecular weight and manageable synthesis with an increased capability to reach solid tumors. ${ }^{65}$

\section{CONCLUSION}

Small molecules have been introduced as a treatment strategy since the past few decades and has been a success so far. The small molecules target the intracellular pathways which deregulate the signaling mechanisms of cancer progression. They act on multiple proteins which play important roles in cancer cell survival and proliferation. In this review, small molecules are classified into chemical moieties: quinoline, quinazoline, pyridine, imidazole and indole and these moieties have various mechanisms of action i.e. inhibition of VEGFR-2 receptors, tyrosine kinases, HER-2 receptor activity etc. These molecules also affect by initiating apoptotic caspases. A single molecule has its effect over several critical cancer targets which brings in the advantages of polypharmacology that brings in a simplified treatment regimen, target specificity, oral administration and better patient compliance. The rate of innovation in anti-cancer treatment comes at a very slow pace. This is leading to an urgent requirement for recent advances as the cancer death rate in middle and lower economy countries has reached more than $60 \%$. Further research on small molecules should focus on novel cancer pathways and their drug targets along with an attempt made to improve the drug efficacy.

\section{ACKNOWLEDGEMENT}

The authors appreciate and acknowledge the immense support given by the Management of Birla Institute of Technology, Mesra (Ranchi, India) and thank for the facilities provided.

\section{CONFLICT OF INTEREST}

The authors declare no conflicts of interest.

\section{ABBREVIATIONS}

ER: Estrogen Receptor; PR: Progesterone Receptor; EGFR: Epidermal Growth Factor Receptor; HER: Human Epidermal growth factor Receptor; TNBC: Triple Negative Breast Cancer; MBC: Metastatic Breast Cancer; HDAC: Histone Deacetylase; MMPs: Matrix Metalloprotease; HSPs: Heat Shock Proteins; SMPP: Small Molecule PolyPharmacology; SMDC: Small Molecule Drug Conjugate; VEGFR: Vascular Endothelial Growth Factor Receptors.

\section{REFERENCES}

1. Kaboli PJ, Rahmat A, Ismail P, Ling $\mathrm{KH}$. Targets and mechanisms of berberine, a natural drug with potential to treat cancer with special focus on breast cancer. European Journal of Pharmacology. 2014;740:584-95.

2. Yeo SK, Guan JL. Breast cancer: Multiple subtypes within a tumor?. Trends in cancer. 2017;3(11):753-60.

3. Ihemelandu CU, Lefall JL, Dewitty RL, Naab TJ, Mezghebe HM, Makambi KH, et al. Molecular breast cancer subtypes in premenopausal and postmenopausal African-American women: Age-specific prevalence and survival. Journal of Surgical Research. 2007;143(1):109-18.

4. Su K, Hu P, Wang X, Kuang C, Xiang Q, Yang F, et al. Tumor suppressor berberine binds VASP to inhibit cell migration in basal-like breast cancer. Oncotarget. 2016;7(29):458-9.

5. Carey LA, Perou CM, Livasy CA, Dressler LG, Cowan D, Conway K, et al. Breast cancer subtypes and survival in the Carolina Breast Cancer Study. Jama. 2006;295(21):2492-502.

6. Cheang MC, Chia SK, Voduc D, Gao D, Leung S, Snider J, et al. Ki67 index, HER2 status and prognosis of patients with luminal B breast cancer. Journal of the National Cancer Institute. 2009;101(10):736-50.

7. Hassan MSU, Ansari J, Spooner D, Hussain SA. Chemotherapy for breast cancer (review). Oncology Reports. 2010;24(5):1121-31.

8. Jänicke F, Prechtl A, Thomssen C, Harbeck N, Meisner C, Untch M, et al. Randomized adjuvant chemotherapy trial in high-risk, lymph node-negative breast cancer patients identified by urokinase-type plasminogen activator and plasminogen activator inhibitor type 1 . Journal of the National Cancer Institute. 2001;90(12):913-20.

9. Duffy MJ, Crown J. A personalized approach to cancer treatment: How biomarkers can help. Clinical Chemistry. 2008;54(11):1770-9.

10. Macielag MJ. Chemical properties of antimicrobials and their uniqueness. Antibiotic Discovery and Development. 2012;793-820.

11. Lavanya V, Mohamed AA, Neesar A, Arun KR, Shazia J. Small molecule inhibitors as emerging cancer therapeutics. Integr Cancer Sci Ther. 2014;1(3):39-46.

12. Lindberg RA, Quinn A, Hunter T. Dual-specificity protein kinases: Will any hydroxyl do?. Trends in Biochemical Sciences. 1992;17(3):114-9.

13. Yarden $Y$, Sliwkowski M. Untangling the ErbB signalling network. Nature Reviews: Molecular Cell Biology. 2001;2(2):127-37.

14. Chaffer CL, Weinberg R. A perspective on cancer cell metastasis. Science. 2011;331(6024):1559-64. 
15. Tebbutt N, Pedersen M, Johns TG. Targeting the ERBB family in cancer: Couples therapy. Nature Reviews Cancer. 2013;13(9):663-73.

16. Lin NU, Winer EP. New targets for therapy in breast cancer: Small molecule tyrosine kinase inhibitors. Breast Cancer Research. 2004;6(5):204-10.

17. Levitzki A, Mishani E. Tyrphostins and other tyrosine kinase inhibitors. Annu Rev Biochem. 2006;75:93-109.

18. Giordano S, Petrelli A. From single-to multi-target drugs in cancer therapy: when aspecificity becomes an advantage. Current Medicinal Chemistry. 2008;15(5):422-32.

19. Anighoro A, Bajorath J, Rastelli G. Polypharmacology: Challenges and opportunities in drug discovery: Miniperspective. Journal of Medicinal Chemistry. 2014;57(19):7874-87.

20. Bolognesi ML. Polypharmacology in a single drug: Multitarget drugs. Current Medicinal Chemistry. 2013;20(13):1639-45.

21. Rosini M. Polypharmacology: The rise of multitarget drugs over combination therapies. Future Medicinal Chemistry. 2014;6(5):485-7.

22. O'Neill PM, Ward SA. A Quinoline Carboxamide Antimalarial Drug Candidate Uniquely Targets Plasmodia at Three Stages of the Parasite Life Cycle. Angewandte Chemie International Edition. 2015;54(46):13504-6.

23. Bharathkumar H, Mohan CD, Ananda H, Fuchs JE, Li F, Rangappa S, et al. Microwave-assisted synthesis, characterization and cytotoxic studies of novel estrogen receptor a ligands towards human breast cancer cells. Bioorganic and Medicinal Chemistry Letters. 2015;25(8):1804-7.

24. Leung E, Hung JM, Barker D, Reynisson J. The effect of a thieno [2, 3-b] pyridine PLC- $y$ inhibitor on the proliferation, morphology, migration and cell cycle of breast cancer cells. Med Chem Comm. 2014;5(1):99-106.

25. Yin L, Hu Q, Hartmann RW. Tetrahydropyrroloquinolinone type dual inhibitors of aromatase/aldosterone synthase as a novel strategy for breast cancer patients with elevated cardiovascular risks. Journal of Medicinal Chemistry. 2013;56(2):460-70.

26. Jain S, Chandra V, Jain PK, Pathak K, Pathak D, Vaidya A. Comprehensive review on current developments of quinoline-based anticancer agents. Arabian Journal of Chemistry. 2016.

27. Liang KW, Ting CT, Yin SC, Chen YT, Lin SJ, Liao JK, et al. Berberine suppresses MEK/ERK-dependent Egr-1 signaling pathway and inhibits vascular smooth muscle cell regrowth after in vitro mechanical injury. Biochemical Pharmacology. 2006;71(6):806-17.

28. Lin CM, Chen CS, Chen CT, Liang YC, Lin JK. Molecular modeling of flavonoids that inhibits xanthine oxidase. Biochemical and Biophysical Research Communications. 2002;294(1):167-72.

29. Tomicic MT, Kaina B. Topoisomerase degradation, DSB repair, p53 and IAPS in cancer cell resistance to camptothecin-like topoisomerase I inhibitors. Biochimica Et Biophysica Acta (BBA)-Reviews on Cancer. 2013;1835(1):11-27.

30. Eisenberg LA, Bialik S, Simon HU, Kimchi A. Life and death partners: Apoptosis, autophagy and the cross-talk between them. Cell Death and Differentiation. 2009;16(7):996.

31. Vrba J, Dolezel P, Vičar J, Modrianský M, Ulrichová J. Chelerythrine and dihydrochelerythrine induce $\mathrm{G} 1$ phase arrest and bimodal cell death in human leukemia HL-60 cells. Toxicology in vitro. 2008;22(4):1008-17.

32. Pan X, Han H, Wang L, Yang L, Li R, Li Z, et al. Nitidine chloride inhibits breast cancer cells migration and invasion by suppressing c-Src/FAK associated signaling pathway. Cancer Letters. 2011;313(2):181-91.

33. Park SY, Jin ML, Kim YH, Lee SJ, Park G. Sanguinarine inhibits invasiveness and the MMP 9 and COX 2 expression in TPA-induced breast cancer cells by inducing HO-1 expression. Oncology Reports. 2014;31(1):497-504.

34. Alagarsamy V, Chitra K, Saravanan G, Solomon VR, Sulthana MT, Narendhar B. An overview of quinazolines: Pharmacological significance and recent developments. European Journal of Medicinal Chemistry. 2018;151:628-85.

35. Panja SK. Anti-cancer therapeutic potential of quinazoline based small molecules via global upregulation of miRNAs. Chemical Communications. 2014;50(35):4639-42.

36. Chinnaiyan P, Huang S, Vallabhaneni G, Armstrong E, Varambally S, Tomlins $\mathrm{SA}$, et al. Mechanisms of enhanced radiation response following epidermal growth factor receptor signaling inhibition by erlotinib (Tarceva). Cancer Research. 2005;65(8):3328-35.

37. Blackwell KL, Burstein HJ, Storniolo AM, Rugo H, Sledge G, Koehler M, et al. Randomized study of Lapatinib alone or in combination with trastuzumab in women with ErbB2-positive, trastuzumab-refractory metastatic breast cancer. Journal of Clinical Oncology. 2010;28(7):1124-30

38. Green MD, Francis PA, Gebski V, Harvey V, Karapetis C, Chan A, et al. Gefitinib treatment in hormone-resistant and hormone receptor-negative advanced breast cancer. Annals of Oncology. 2009;20(11):1813-7.

39. Smaill JB, Palmer BD, Rewcastle GW, Denny WA, McNamara DJ, Dobrusin EM, et al. Tyrosine kinase inhibitors: 15. 4-(Phenylamino) quinazoline and 4-(phenylamino) pyrido [d] pyrimidine acrylamides as irreversible inhibitors of the ATP binding site of the epidermal growth factor receptor. Journal of Medicinal Chemistry. 1999;42(10):1803-15.

40. Hoehn D, Cortes JE, Medeiros LJ, Jabbour EJ, Hidalgo JE, Kanagal SR, et al. Multiparameter Analysis of Off-Target Effects of Dasatinib on Bone Homeostasis in Patients With Newly Diagnosed Chronic Myelogenous Leukemia. Clinical Lymphoma Myeloma and Leukemia. 2016;16(Suppl):86-92.

41. Davari AS, Abnous K, Mehri S, Ghandadi M, Hadizadeh F. Synthesis and biological evaluation of novel pyridine derivatives as potential anticancer agents and phosphodiesterase-3 inhibitors. Bioorganic Chemistry. 2014;57:83-9.

42. Choi HE, Yoo MS, Choi JH, Lee JY, Kim JH, Kim JH, et al. BRN-103, a novel nicotinamide derivative, inhibits VEGF-induced angiogenesis and proliferation in human umbilical vein endothelial cells. Bioorganic and Medicinal Chemistry Letters. 2011;21(21):6236-41.

43. Wilhelm S, Carter C, Lynch M, Lowinger T, Dumas J, Smith RA, et al. Discovery and development of sorafenib: A multikinase inhibitor for treating cancer. Nature Reviews Drug Discovery. 2006;5(10):835.

44. Solomon BJ, Mok T, Kim DW, Wu YL, Nakagawa K, Mekhail T, et al. First-line crizotinib versus chemotherapy in ALK-positive lung cancer. New England Journal of Medicine. 2014;371(23):2167-77.

45. Ponzo MG, Lesurf R, Petkiewicz S, O'Malley FP, Pinnaduwage D, Andrulis IL, et al. Met induces mammary tumors with diverse histologies and is associated with poor outcome and human basal breast cancer. Proceedings of the National Academy of Sciences. 2009;106(31):12903-8.

46. Ali I, Lone MN, Aboul-Enein HY. Imidazoles as potential anticancer agents. Med Chem Comm. 2017;8(9):1742-73.

47. Tolcher AW, Gerson SL, Denis L, Geyer C, Hammond LA, Patnaik A, et al. Marked inactivation of $O$ 6-alkylguanine-DNA alkyltransferase activity with protracted temozolomide schedules. British Journal of Cancer. 2003;88(7):1004

48. Amé JC, Rolli V, Schreiber V, Niedergang C, Apiou F, Decker P, et al. PARP-2, A novel mammalian DNA damage-dependent poly (ADP-ribose) polymerase. Journal of Biological Chemistry. 1999;274(25):17860-8.

49. Rouleau M, Patel A, Hendzel MJ, Kaufmann SH, Poirier GG. PARP inhibition: PARP1 and beyond. Nature Reviews Cancer. 2010;10(4):293.

50. Jiang HL, Sun HF, Gao SP, Li LD, Huang S, Hu X, et al. SSBP1 suppresses TGF $\beta$-driven epithelial-to-mesenchymal transition and metastasis in triplenegative breast cancer by regulating mitochondrial retrograde signaling. Cancer Research. 2016;76(4):952-64.

51. Jia X, Hong Q, Lei L, Li D, Li J, Mo M, et al. Basal and therapy-driven hypoxia-inducible factor-1a confers resistance to endocrine therapy in estrogen receptor-positive breast cancer. Oncotarget. 2015;6(11):8648.

52. Zafarnia S, Ibach JB, Spivak I, Li Y, Koletnik S, Doleschel D, et al. Nilotinib enhances tumor angiogenesis and counteracts VEGFR2 blockade in an orthotopic breast cancer xenograft model with desmoplastic response. Neoplasia. 2017;19(11):896-907.

53. Tanaka T, Ikegami Y, Nakazawa H, Kuriyama N, Oki M, Hanai JI, et al. LowDose Farnesyltransferase Inhibitor Suppresses HIF-1a and Snail Expression in Triple-Negative Breast Cancer MDA-MB-231 Cells in vitro. Journal of Cellular Physiology. 2017;232(1):192-201.

54. Ahmad A, Sakr WA, Rahman KM. Mechanisms and therapeutic implications of cell death induction by indole compounds. Cancers. 2011;3(3):2955-74

55. Fischer E, Jourdan F. Ueber die hydrazine der brenztraubensäure. Berichte Der Deutschen Chemischen Gesellschaft. 1883;16(2):2241-5.

56. Johnson IS, Armstrong JG, Gorman M, Burnett JP. The vinca alkaloids: A new class of oncolytic agents. Cancer Research. 1963;23(8). 
57. Kumar S, Tomar MS, Acharya A. PKC-Inhibitors Induce Apoptosis by Inhibiting Hsp70-Mediated Protection of Anti-Apoptotic Bcl ${ }_{-2}$ Proteins in Dalton's Lymphoma Cells. Journal of Tumor. 2016;4(3):435-44.

58. Chow LQ, Eckhardt SG. Sunitinib: From rational design to clinical efficacy. Journal of Clinical Oncology. 2007;25(7):884-96.

59. JrSledge GW, Polar YG. Protein kinase C- $\beta$ as a therapeutic target in breast cancer. Seminars in Oncology. 2006;33(3 Suppl 9):15-8.

60. Liang XJ, Chen C, Zhao Y, Wang PC. Humana Press. Circumventing tumor resistance to chemotherapy by nanotechnology. Multi Drug Resistance in Cancer. 2010;596:467-88.

61. Shakeri A, Sahebkar A. Opinion Paper: Nanotechnology: A Successful Approach to Improve Oral Bioavailability of Phytochemicals. Recent Patents on Drug Delivery and Formulation. 2016;10(1):4-6.
62. Jin $\mathrm{H}, \mathrm{Pi}$ J, Zhao Y, Jiang J, Li T, Zeng X, et al. EGFR-targeting PLGA-PEG nanoparticles as a curcumin delivery system for breast cancer therapy. Nanoscale. 2017;9(42):16365-74.

63. Deng ZJ, Morton SW, Ben-Akiva E, Dreaden EC, Shopsowitz KE, Hammond PT. Layer-by-layer nanoparticles for systemic codelivery of an anticancer drug and si RNA for potential triple-negative breast cancer treatment. ACS Nano. 2013;7(11):9571-84.

64. Eloy JO, DeSouza MC, Petrilli R, Barcellos JP, Lee RJ, Marchetti JM. Liposomes as carriers of hydrophilic small molecule drugs: Strategies to enhance encapsulation and delivery. Colloids and Surfaces B: Biointerfaces. 2014;123:345-63.

65. Zhuang C, Guan X, Ma H, Cong H, Zhang W, Miao Z. Small molecule-drug conjugates: A novel strategy for cancer-targeted treatment. European Journal of Medicinal Chemistry. 2018;163:883-95.
PICTORIAL ABSTRACT

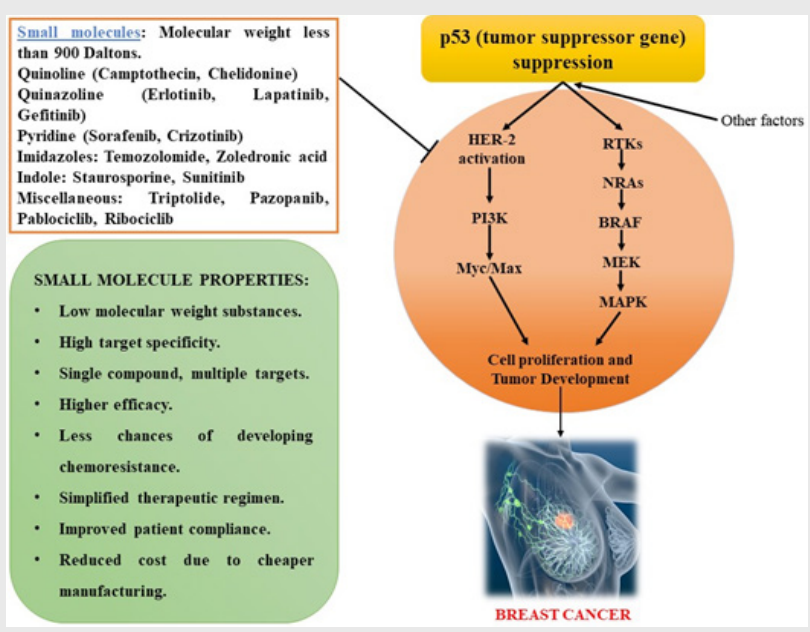

\section{SUMMARY}

- Breast cancer is a major problem all around the globe. There is a desperate need for new innovations for the treatment of breast cancer.

- The emerging trend in the treatment of cancer is small molecules which have very less molecular weight and shows their target specificity at cellular levels.

- The review focused on emerging new small molecules and their multitarget action towards various receptors which could be a useful weapon in the future in the combat against breast cancer.

\section{About Authors}

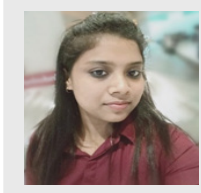

Reetuparna Acharya is a Research Scholar from Birla Institute of Technology, Mesra, Ranchi and she is currently pursuing her Ph.D. on small molecules synthesis and evaluation of their molecular mechanistic pathway on mammary carcinogenesis. She had finished her Master's degree in Pharmacology with the molecular evaluation of the anticancer mechanism of quinazoline derivatives on breast cancer model in Sprague Dawley rats.

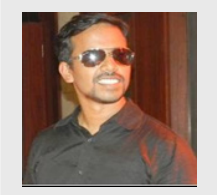

Dr. Shakti Prasad Pattanayak is currently working as an Assistant Professor (AL 12) in the Division of Pharmacology, Birla Institute of Technology Mesra (BIT), Ranchi, India since last 12 years. He had worked as a postdoctoral researcher at Faculty of Medicine, The Hebrew University of Jerusalem, Israel, which is a home University to Sir Albert Einstein. His research is related to advanced functional mammary carcinogenesis with molecular pathways such as mTOR, LXR $\alpha$, Nrf2/Keap 1 etc. signaling. He had worked with eminent scientists like Prof. Boaz Tirosh (mTOR Signaling), Dr. Galia Blum (Cathepsin B), Dr. Michel Berger (FACS and Cell-sorting) (Lautenberg Center for Immunology and Cancer Research). He has been awarded with PBC award as an outstanding postdoctoral researcher from India and China.

Cite this article: Acharya R, Pattanayak SP. Small Molecules Polypharmacology for the Treatment of Breast Cancer: A Roadmap for the Future Chemotherapy. Indian J of Pharmaceutical Education and Research. 2020;54(2):225-33. 